\title{
Extracting Blink Rate Variability from EEG Signals
}

\author{
Temesgen Gebrehiwot, Rafal Paprocki, Marija Gradinscak, Artem Lenskiy
}

\begin{abstract}
Generally, blinks are treated on equal with artifacts and noise while analyzing EEG signals. However, blinks carry important information about mental processes and thus it is important to detect blinks accurately. The aim of the presented study is to propose a blink detection method and discuss its application for extracting blink rate variability, a novel concept that might shed some light on the mental processes. In this study, 14 EEG recordings were selected for assessing the quality of the proposed blink detection algorithm.
\end{abstract}

Index Terms-Blink rate variability, inter blink interval dynamics, EEG artifacts.

\section{INTRODUCTION}

Blinking is a semi-autonomic closing of the eye lids. Blinks keep eyes protected against potentially damaging stimuli, such as bright lights and foreign bodies like dust. The sudden changes in an image due to saccades or blinks does not interfere with our subjective experience of continuity [1]. The very act of blinking suppresses activity in several areas of the brain responsible for detecting environmental changes, so that one experiences the world as continuous. Researchers have shown synchronous behavior in blinking between listener and speaker in face-to-face conversation [2]. Reduced blink rate causes eye redness and dryness also known as Dry Eye, which is a major symptom of the Computer Vision Syndrome [3]. Blinks have been known to be linked to interior brain activities. Increasing the accuracy of blink detection is of high importance as humans look for easier methods of collecting internal brain activity information. The detection of the eye blinks had a huge impact in various fields. In some Brain Computer Interface (BCI) researchers analyzed eye blinks to determine the pattern and the duration between blinks. After collecting this analysis, the information was used with a device that could control a computer similarly to how we use a computer mouse. This implementation of the use of blinks has opened a wide door to new possibilities for disabled people [4]. Another area where blinks play an important role is in the prevention of car accidents. The World Health Organization (WHO) has announced that the ninth cause of death, globally, is car accidents. The National Motor Vehicle Crash Causation Survey (NMVCCS) has found that 30 percent of car accidents happen due to the drowsiness of drivers [5]. It is noted that workload increases blink rate and blink rate is known to decrease in monotonous and drowsy conditions [6]. Blink rate $(\mathrm{BR})$ is inversely correlated with the increase of

Manuscript received November 12, 2015; revised June 1, 2016.

Artem Lenskiy is with Korea University of Technology and Education 1600, Chungjeol-ro, Byeongcheon-myeon, Dongnam-gu, Cheonan-si, Chungcheongnam-do 31253, Republic of Korea (e-mail: lensky@koreatech.ac.kr). workload so blinks can be used to detect drowsiness before it causes damage [6]. Researchers have shown that blinks can play a significant role in detecting many different brain disorders and brain activities. Spontaneous BR has been studied in many neurological diseases like Parkinson's disease and Tourette syndrome [7]-[9]. The use of blink detection does not stop there. Blinks are regarded as a non-invasive peripheral markers of central dopamine activity which makes their accurate detection more important [10]-[15]. Researchers have studied the synchronicity of the eye blinks in audiences, who experienced the same method of storytelling. The eye blink synchronization among audiences is driven by attention cycles, which are in turn driven by emotional processing [16]-[18]. Blinks are not always the most desired signals when it comes to non-invasive brain signal measuring as many electroencephalographs (EEG) remove them to acquire brain data. Eye blink is one of the main artifacts in the EEG signals [19]. Researchers are focusing on removing these parts of the signals to obtain clean brain signal values. To analyze blinks and variation of inter-blink intervals it is important to detect blinks accurately. We propose to apply the blink detection algorithm for extracting the inter-blink intervals that we coin the blink rate variability (BRV) in analogy to heart rate variability. We construct BRV for subjects taking memory tests. We further compare the numbers of detected blinks by the algorithm and by manual counting.

\section{PROCEDURE FOR PAPER SUBMISSION}

\section{A. Data Acquisition}

The video stream was captured with a Pointgrey Flea3 USB camera. The video stream was stored on a disk drive to be processed in the future. Simultaneously, EEG signals were recorded. For the recording of EEG signals, we employed a Mitsar-EEG 201 amplifier and used WinEEG software. The electrodes were placed according to the international 10-20 system [20]. Electro-gel was injected into electrodes' hollow in order to decrease the electrode-skin resistance. Currently, the EEG signals were recorded for the purpose of eye blink detection. In the future, we are planning to analyze EEG to detect various types of brain activity. The experimental setup is shown in Fig. 1.

\section{B. Testing Procedures}

The testing software was developed using Java in such a way that it does not require any interventions. The procedure consisted of a five minute reading session and a five minute memory test session. Before the memory test, a passage about Ethiopia is given. After reading the passage, users are presented questions one by one. In this paper, we focus on detecting blinks while subjects are reading the passage and 
answering questions about the passage.

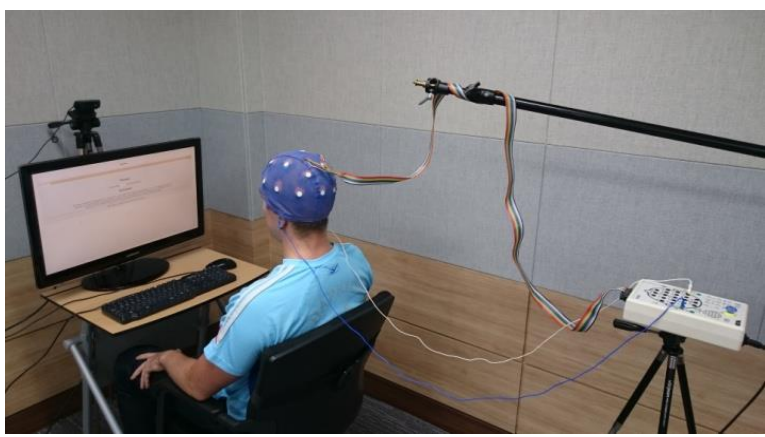

Fig. 1. Experimental setup.

Overall, 28 people participated in the experiment. Among them 14 subjects were dropped due to falling asleep, adjusting the cap or constant head movements that resulted in significant noise.

\section{Eye BLINK DETECTION PROCEDURE}

Electrodes are applied to the head according to the 10-20 system. We used bipolar montage, which means we determined the potential between Fp1 and Fp3, along with Fp2 and Fp4. Fig. 2 presents EEG signals for both pairs. EEG signals were recorded while participants were taking the tests and imported in form of CSV files to Matlab(c) for further analysis. The process of blink detection can be divided into two stages: the preprocessing (algorithm 1) and the blink detection (algorithm 2). The preprocessing stage consists of the following steps: (a) normalization and bandpass filtering (b) cutting of extreme amplitudes using the estimated cumulative distribution function that characterizes the signal amplitude's distribution, (c) independent component analysis, and (d) selection of the component with eye blinks. The blink detection stage consists of (e) signal thresholding, (f) candidate extraction, and $(\mathrm{g})$ polynomial fitting with finding maximum in the polynomial function. Both stages, preprocessing and blink detection, have been presented in listings algorithm 1 and algorithm 2 respectively. The first step in the process of blink detection consists of cutting off very extreme amplitudes, that usually caused by touching the electrodes or cap adjustment. Right after, the signal is band-pass filtered and normalized. We applied 50th-order bandpass filter with finite impulse response. The lowest and highest normalized cut off frequencies are $f_{L}=0.02$ and $f_{H}=$ 0.08 correspondingly. In fig. 3 the EEG signals after bandpass filtering and normalization are presented. The signals became smoother and the lower frequency components responsible for trends in the signals disappear after filtering. For the filtered signal a cumulative distribution function $(\mathrm{CDF})$ is estimated. Using the $\mathrm{CDF}$, we cut 2 percent of all amplitudes from the top and 1 percent from the bottom. The next step consists of mixing signals from two pairs of electrodes fp1-fp3 and fp2-fp4 in such a way that led to a cleaner signal.

Usually, we want to get rid of ocular artifacts from EEG signals, as the eye blink is an artifact and leads to interpretation problems [21]. However, our goal is on contrary aims at extracting blinks from EEG. We employ the fastICA [22] algorithm for solving Blind Source Separation
(BSS) [23], which allows us to differentiate neural activity from muscle and blinks [24].

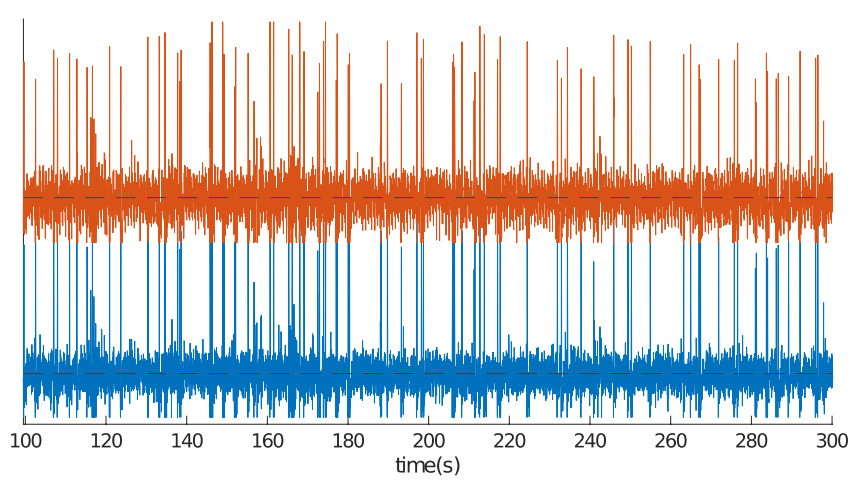

Fig. 2. Original Fp1-Fp3 and Fp2-Fp4 electrode pairs.
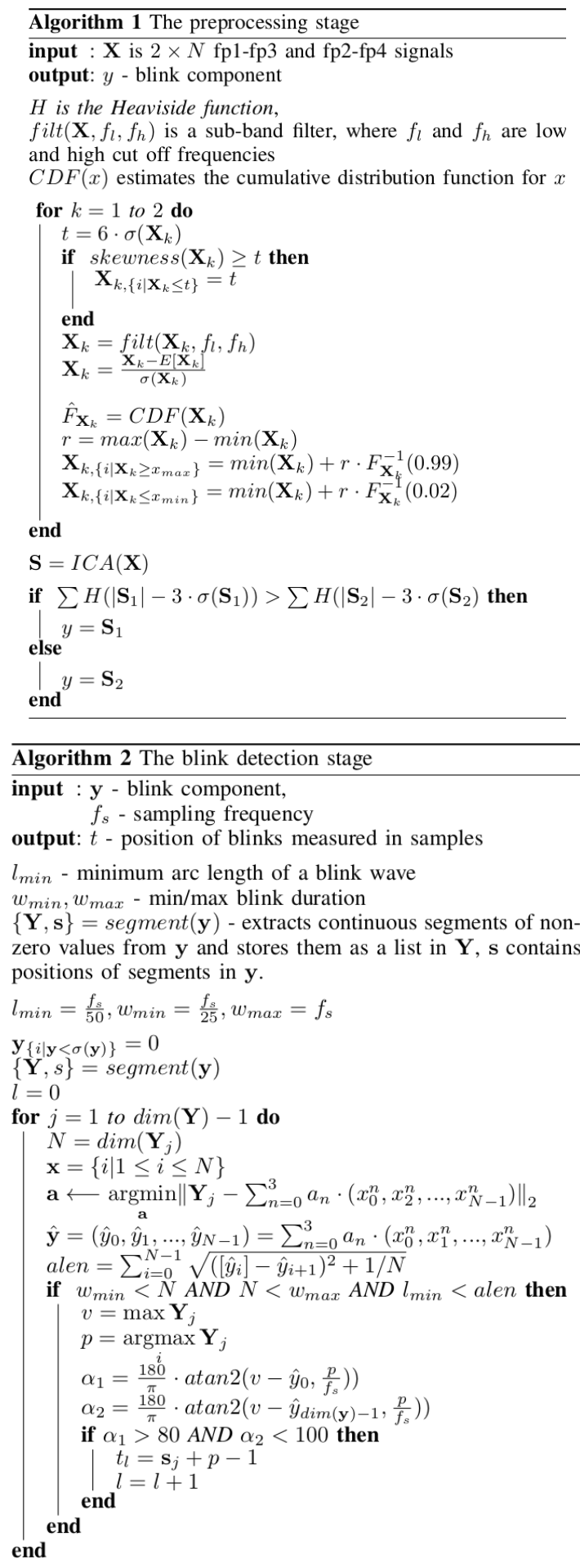


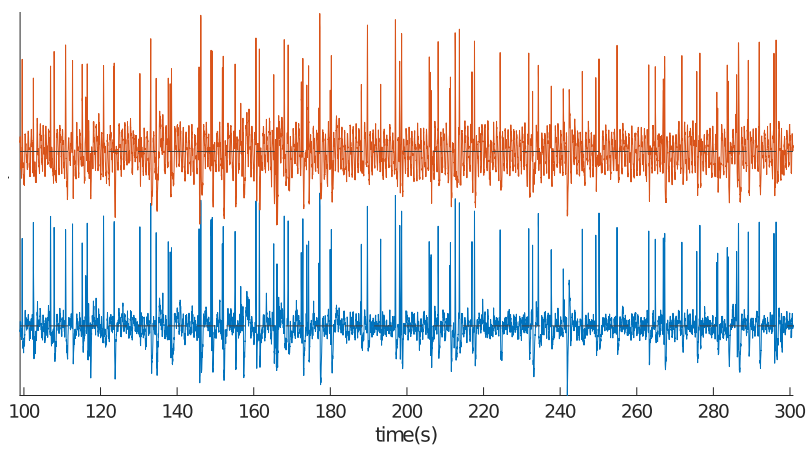

Fig. 3. Original Fp1-Fp3 and Fp2-Fp4 electrode pairs.

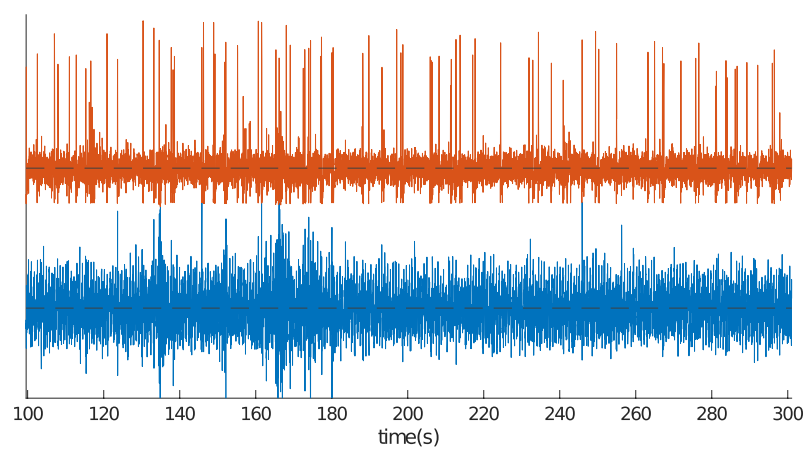

Fig. 4. Independent components.

Independent component analysis (ICA) consists of two steps. The first is responsible for decorrelation or whitening, when a correlation in the data is removed. The second stage is responsible for the separation, which is an orthogonal transformation of whitened signals (rotation of the joint density). The task is to find an orthogonal matrix $U$ such that the projections on the orthogonal axis are non- Gaussian [22]. One by one, we look for the rows of the matrix $U=$ $\left(u_{1}, u_{2}\right)^{T}$ so a measure of non-Gaussianity $\left|E\left(G\left(u_{k}^{T} x_{s t}\right)\right)\right|$ is maximized by such $u_{k}$ that the length of $u_{k}$ is one and orthogonal to the remain row. The function $G$ can be any nonquadratic function, which is twice continuously differentiable with $G(0)=0$. We tested a number of nonlinear functions and the one that results in a better component separation is the $g(z)=z^{2}$ skewness measure. The $g(z)=z^{2}$ (skew) nonlinearity finds skew sources, but in the case of symmetric sources is not efficient. In our data, the skew measure shows best results due to the fact that the waveforms corresponding to blinks are asymmetrical (Fig. 4). To distinguish which of the components corresponds to the blink component $y$, we select the component based on the following rule

$$
y=\left\{\begin{array}{cc}
\sum H\left(\left|S_{1}\right|-\sigma_{3}\left(S_{1}\right)\right)>\sum_{\text {otherwise }} H\left(\left|S_{2}\right|-\sigma\left(S_{2}\right)\right), & \text { blinks } \\
\text { noise }
\end{array}\right.
$$

where $H$ is the Heaviside function. In the formula above we count a number of crossings at $\sigma_{3}=3 * \sigma\left(S_{i}\right)$. At the next step, we set to zero all samples that are smaller than the standard deviation of the signal. The samples below the threshold are zeroed. The remaining contiguous segments are treated as blink candidates (Fig. 4). Finally, a $3^{\text {rd }}$ order polynomial function is fitted to the samples within each segment. If the arc length of the polynomial function is less than a predefined threshold, the region is rejected (Fig. 5). We also reject regions with a slop of the front and the end transitions having an angle less than 80 degrees for the front and having greater than 100 degrees for the end transition. The slop is calculated as an angle of line connecting end points of the fitting polynomial and its maximum (Fig. 6). To construct blink rate variability, the time of occurrences of consecutive blinks are subtracted. The interval between blinks is stack-up into a series that constitutes blink rate variability (Fig. 7).

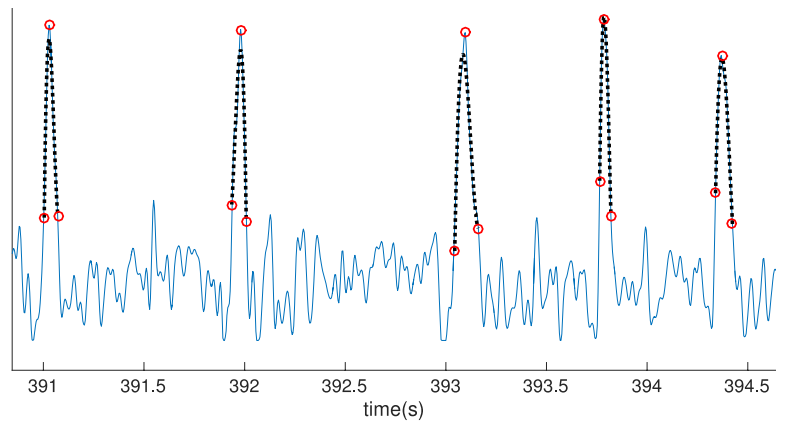

Fig. 6. Detected blinks.

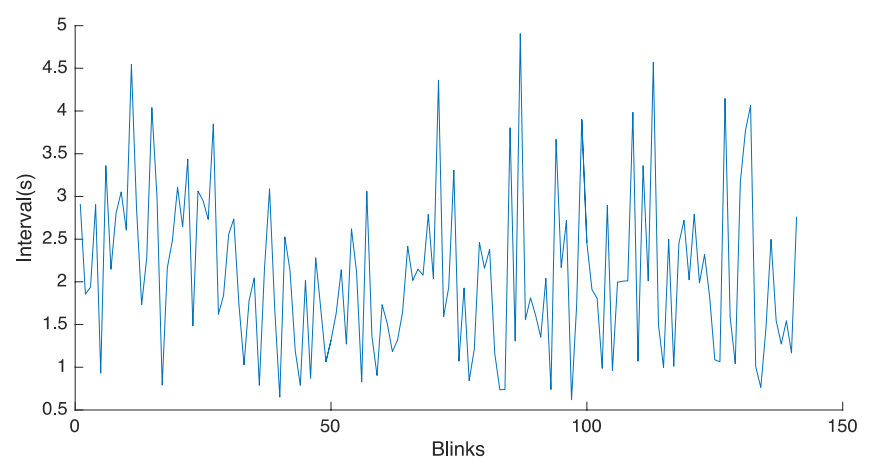

Fig. 7. Example of blink rate variability.

\section{RESUlTS AND DisCUSSION}

Fig. 8 demonstrates BRVs for all of the subjects during the memory test. The abscissa is the blink interval and the ordinate represents the length of inter-blink intervals. The BRV shows the dynamics of blink intervals extracted while memory testing for each subject. To access the quality of blink detection we manually calculated the number of false positives, false negatives and true positives during the reading and the testing stages. The false positives are mistakenly detected blinks at places where blinks did not occur.

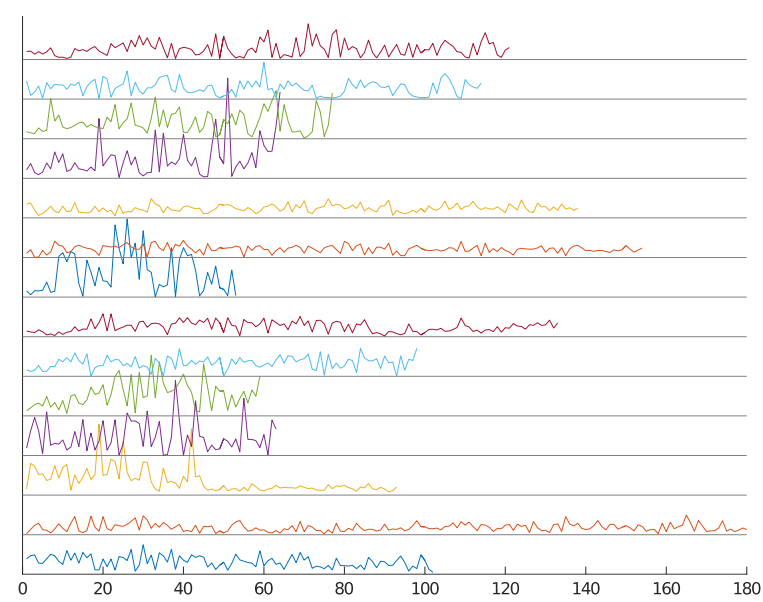

Fig. 8. Extracted blink rate variability for each subject during reading. 
The false negatives are the blinks that were missed, and the true positives are the correctly detected blinks. Based on these three categories we calculated the precision and recall characteristics that are shown in Tables I and II. The average precision during reading stage is $0.99 \pm 0.02$ and the average of recall is $0.99 \pm 0.01$. The average precision during memory testing stage is $0.98 \pm 0.03$ and the average of recall is $0.99 \pm 0.02$.

TABLE I: COMPARISON OF AUTOMATIC AND MANUAL BLINK DETECTION DURING READING STAGE

\begin{tabular}{llllll}
\hline Subject & $\begin{array}{l}\text { False } \\
\text { positive }\end{array}$ & $\begin{array}{l}\text { False } \\
\text { negative }\end{array}$ & $\begin{array}{l}\text { True } \\
\text { positive }\end{array}$ & Precision & Recall \\
\hline 1 & 3 & 0 & 100 & 0.97 & 1.00 \\
2 & 0 & 3 & 180 & 1.00 & 0.98 \\
3 & 0 & 0 & 94 & 1.00 & 1.00 \\
4 & 0 & 0 & 61 & 1.00 & 1.00 \\
5 & 0 & 0 & 60 & 1.00 & 1.00 \\
6 & 0 & 1 & 94 & 1.00 & 0.99 \\
7 & 1 & 0 & 133 & 0.99 & 1.00 \\
8 & 2 & 1 & 49 & 0.96 & 0.98 \\
9 & 4 & 1 & 148 & 0.97 & 0.99 \\
10 & 0 & 0 & 139 & 1.00 & 1.00 \\
11 & 3 & 0 & 62 & 0.95 & 1.00 \\
12 & 1 & 0 & 76 & 0.99 & 1.00 \\
13 & 2 & 3 & 113 & 0.98 & 0.97 \\
14 & 0 & 1 & 122 & 1.00 & 0.99 \\
\hline
\end{tabular}

TABLE II: COMPARISON OF AUTOMATIC AND MANUAL BLINK DETECTION DURING TESTING STAGE

\begin{tabular}{llllll}
\hline Subject & $\begin{array}{l}\text { False } \\
\text { positive }\end{array}$ & $\begin{array}{l}\text { False } \\
\text { negative }\end{array}$ & $\begin{array}{l}\text { True } \\
\text { positive }\end{array}$ & Precision & Recall \\
\hline 1 & 0 & 1 & 187 & 1.00 & 0.99 \\
2 & 0 & 3 & 212 & 1.00 & 0.99 \\
3 & 1 & 1 & 76 & 0.99 & 0.99 \\
4 & 3 & 3 & 63 & 0.95 & 0.95 \\
5 & 0 & 2 & 146 & 1.00 & 0.99 \\
6 & 0 & 0 & 124 & 1.00 & 1.00 \\
7 & 0 & 0 & 144 & 1.00 & 1.00 \\
8 & 5 & 0 & 67 & 0.93 & 1.00 \\
9 & 1 & 1 & 197 & 0.99 & 0.99 \\
10 & 3 & 4 & 184 & 0.98 & 0.98 \\
11 & 4 & 0 & 51 & 0.93 & 1.00 \\
12 & 1 & 3 & 88 & 0.99 & 0.97 \\
13 & 0 & 8 & 181 & 1.00 & 0.96 \\
14 & 0 & 0 & 201 & 1.00 & 1.00 \\
\hline
\end{tabular}

\section{CONCLUSION}

Blinking is a natural, biological, semiautomatic process. It is linked to interior brain activity and relationships between blinks and performing tasks. The blink rate variability might find applications in variety of fields, including car safety, psychology or BCI. In order to automatically extract blinks from large datasets of EEG recordings, we proposed the blink detection algorithm. Basic steps such as band-pass filtering and thresholding are applied in the algorithm. Then fastICA is applied to find an independent component within the blinks. The blink candidates were filtered based on the heuristics that arc length of the blink waveform should be above a certain threshold and the slopes of the blink waveform are within a $\left[80^{\circ} 100^{\circ}\right]$ range. Calculated the recall and precision characteristics show that the proposed algorithm is suitable for blink rate variability extraction. The Matlab code for blink detection and the data are available online [25].

\section{REFERENCES}

[1] T. J. Gawne and J. M. Martin, "Responses of primate visual cortical neurons to stimuli presented by flash, saccade, blink, and external darkening," J Neurophysiol, 2002.
[2] J. U. Duncombe, "Infrared navigation - Part I: An assessment of feasibility," IEEE Trans. Electron Devices, vol. ED-11, pp. 34-39, Jan. 1959.

[3] T. Nakano and S. Kitazawa, "Eyeblink entrainment at breakpoints of speech," Exp. Brain Res., 2010.

[4] P. Polatsek, Eye Blink Detection, Slovak University of Technology, Bratislava, April 23, 2013, p. 18.

[5] M. Chau and M. Betke, Real Time Eye Tracking and Blink Detection with USB Cameras, Computer Science Department Boston University Boston, MA 02215, USA, National Motor Vehicle Crash Causation Survey(NMVCCS).

[6] G. Barbato, G. Ficca, G. Muscettola, M. Fichele, M. Beatrice, and F. Rinaldi, "Diurnal variation in spontaneous eye-blink rate," Psychiatry Res., 2000, vol. 93, pp. 145-151

[7] R. Agostino, A. Berardelli, G. Cruccu, F. Stocchi, and M. Manfredi, "Corneal and blink reflexes in Parkinsons disease with onoff fluctuations," Mov. Disord., vol. 2, pp. 227-235, 1987.

[8] J. Shlik, Y. Zhou, D. Koszycki, F. J. Vaccarino, and J. Bradwejn, "Effects of CCK-4 infusion on the acoustic eye-blink startle and psychophysiological measures in healthy volunteers," $J$. Psychopharmacol., vol. 13, pp. 385-390, 1999.

[9] C. N. Karson, C. A. Kaufmann, A. K. Shapiro, and E. Shapiro, "Eye-blink rate in Tourettes syndrome," J. Nerv. Ment. Dis., vol. 173 , pp. 555-559, 1985.

[10] E. Y. Chen, L. C. Lam, R. Y. Chen, and D. G. Nguyen, "Blink rate, neurocognitive impairments, and symptoms in schizophrenia," Biol. Psychiatry, vol. 40, pp. 597-503, 1995.

[11] P. M. Helms and C. D. Godwin, "Abnormalities of blink rate in psychoses: A preliminary report," Biol. Psychiatry, vol. 20, pp. 103-105, 1985.

[12] A. Mackert, C. Woyth, K. M. Flechtner, and H. P. Volz, "Increased blink rate in drug-naive acute schizophrenic patients," Biol. Psychiatry, vol. 27, pp. 1197-1202, 1990.

[13] R. Sandyk, "The significance of eye blink rate in parkinsonism: A hypothesis," Int. J. Neurosci., vol. 51, pp. 99-103, 1990.

[14] L .K. Jacobsen, D. W. Hommer, and W. L. Wong, "Blink rate in childhood-onset schizophrenia: Comparison with normal and attention-deficit hyperactivity disorder controls," Biol. Psychiatry, vol. 40, 1222-1229, 1995.

[15] C. N. Karson, R. Dykman, S. R. Paige, "Blink rates in schizophrenia," Schizophr. Bull., vol. 15, pp. 345-354, 1990.

[16] R. Nomura, K. Hino, M. Shimazu, Y. Liang, and T. Okada, "Emotionally excited eyeblink-rate variability predicts an experience of transportation into the narrative world".

[17] R. Nomura and T. Okada, "Spontaneous synchronization of eye-blinks during story-telling performance," Cogn. Stud., vol. 21, pp. 225-244, 2014.

[18] T. Nakano, Y. Yamamoto, K. Kitajo, T. Takahashi, and S. Kitazawa, "Synchronization of spontaneous eyeblinks while viewing video stories," in Proc. R. Soc. B Biol. Sci., 2009, vol. 275, pp. 3535-3544.

[19] B. Chambayil, R. Singla, and R. Jha, "EEG Eye Blink Classification Using Neural Network," in Proc. the World Congress on Engineering, 2010, London, U.K.

[20] J. Malmivuo and R. Plonsey, "Bioelectromagnetism, Principles and Applications of Bioelectrical and Biomagnetic Fields, New York, Oxford, Oxford University Press, 1995, p. 258.

[21] R. J. Croft and R. J. Barry, "Removal of ocular artifact from the EEG: A review," Clin. Neurophysiol., vol. 30, p. 519, 2000.

[22] J. Miettinen, K. Nordhausen, H. Oja, and S. Taskinen, "Deflation-based FastICA with adaptive choices of nonlinearities," IEEE Transactions on Signal Processing, 2014.

[23] P. Comon, "Independent component analysis, a new concept?" Signal Processing, vol. 35, pp. 287-314, 1994.

[24] A. J. Bell and T. J. Sejnowski, "An information-maximization approach to blind separation and blind deconvolution," Neural Computation, vol. 7, pp. 1129-1159.

[25] GITHUB. [Online]. https://github.com/Lenskiy/brain-analysis.git

Available:

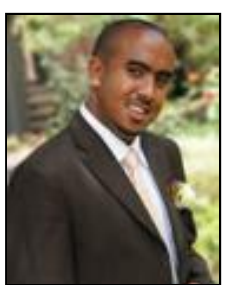

Temesgen Gebrehowt was born in Addis Ababa Ethiopia on December 19, 1988. Mr Gebrehowt went to Hilcoe School of Computer Science and graduated with a bachelor of science in 2011. He recently graduated with the bachelors in communication engineering from Korea University of Technology and Education. 


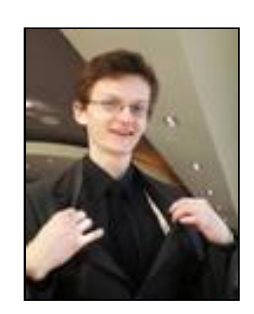

Rafal Paprocki completed the bachelors and the master degrees in Military University of Technology, Warsaw, Poland in 2010 and 2011 respectively. In 2010, Mr Paprocki did an internship at the Università degli Studi di Camerino, Italy. Currently he is a doctor course student at Korea university of Technology and Education, South Korea. His research interest is brain signal processing with applications to inter-blink intervals analysis.

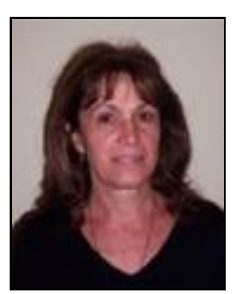

Marija Gradinscak is an assistant professor at the Korea University of Technology and Education, South Korea. From 1996 she has been actively involved in teaching and research. She received her MEng and her Ph.D from Victoria University, Melbourne.

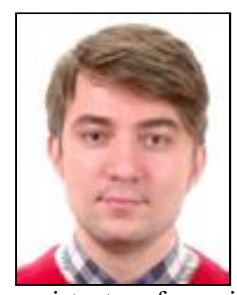

Artem Lenskiy Received BSc and MSc degrees in computer science form Novosibirsk State Technical University, Russian in 2002 and 2004, respectively. After teaching at the same university for a year, he joined doctor course at the University of Ulsan, Korea. He was awarded the Ph.D. in 2010 from the same university. After conducting research as a postdoc fellow at the Ulsan University, he joined Korea University of Technology and Education as an assistant professor in 2011.

His research interests include machine learning and self-similar processing applied to various research and engineering fields including financial time series analysis, telecommunication and physiological signal analysis. 